\title{
Examining the relation of correct knowledge and misconceptions using the nominal response model
}

\author{
John Stewart๑, ${ }^{1,{ }^{*}}$ Byron Drury, ${ }^{2}$ James Wells, ${ }^{3}$ Aaron Adair $\odot,{ }^{2}$ Rachel Henderson, ${ }^{4}$ Yunfei Ma, ${ }^{2}$ \\ Ángel Pérez-Lemonche, ${ }^{2}$ and David Pritchard ${ }^{2}$ \\ ${ }^{1}$ Department of Physics and Astronomy, West Virginia University, \\ Morgantown, West Virginia 26506, USA \\ ${ }^{2}$ Department of Physics, Massachusetts Institute of Technology, Cambridge, Massachusetts 02139, USA \\ ${ }^{3}$ Department of Physics, University of Connecticut, Storrs, Connecticut 06269, USA \\ ${ }^{4}$ Department of Physics and Astronomy, Michigan State University, East Lansing, Michigan 48824, USA
}

(Received 31 October 2020; accepted 16 March 2021; published 29 March 2021)

\begin{abstract}
This study reports an analysis of the Force Concept Inventory (FCI) using item response curves (IRC)the fraction of students selecting each response to an item as a function of their total score. Three large samples $(N=9606,4360$, and 1439) of calculus-based physics students were analyzed. These were drawn from three land-grant institutions with very different average FCI post-test scores. A significant number of the response curves for incorrect responses have a maximum at some intermediate value of the total score on the FCI. To analyze the incorrect responses (rather than just the correct response as is typically done), we used Bock's nominal response model (NRM), primarily because it can fit these intermediate maximum curves. The one-dimensional NRM latent ability dimension was strongly correlated $(r=0.99)$ with the latent ability determined by two-parameter logistic (2PL) item response theory applied with correct or incorrect grading even though the correct responses were not identified for the NRM. To understand the relation of Newtonian and non-Newtonian thinking, higher dimensional models were fit. The twodimensional NRM model produced one ability dimension which could be rotated to be highly correlated with the 2PL latent ability $(r \geq 0.98)$ and a second dimension which was most strongly related to misconceptions involving Newton's third law. Cluster analysis was applied to the two latent ability dimensions producing a three-cluster solution where the cluster centroids were very similar for each of the three institutions. The clusters represented three groups of students: Newtonian thinkers still retaining some misconceptions, non-Newtonian thinkers strongly applying Newton's third law misconceptions, and nonNewtonian thinkers weakly applying those misconceptions. The differences between overall FCI scores at the three institutions could be explained by the relative populations of the three clusters.
\end{abstract}

DOI: 10.1103/PhysRevPhysEducRes.17.010122

\section{INTRODUCTION}

The existence of ships, wagons, bridges, aqueducts, cathedrals, and other manmade structures in the 16th century and before shows that people had useful and systematic beliefs about motion, forces, and materials before the intellectual revolutions brought by Galileo and Newton. Especially noteworthy are those codified by Aristotle and systematically refined by many others, particularly the impetus and kinematics schools of the early 14th century. Traditional introductory physics instruction generally ignored such preexisting beliefs. The role of misconceptions in understanding student understanding of

*jcstewart1@mail.wvu.edu

Published by the American Physical Society under the terms of the Creative Commons Attribution 4.0 International license. Further distribution of this work must maintain attribution to the author(s) and the published article's title, journal citation, and DOI. conceptual physics has been an important area of study in physics education research (PER) since the beginning of the field $[1,2]$. In some sense, the unique challenges students' naive theories of motion present to physics instructors suggested the need for discipline-based educational research in physics. Much of the research into misconceptions has been performed using multiple-choice research-based conceptual mechanics instruments: the Force Concept Inventory (FCI) [3] and the Force and Motion Conceptual Evaluation (FMCE) [4]. Since its introduction in 1992, the PER community has explored incorrect student reasoning using pre- and post-instruction scores on the FCI. This methodology has allowed the convincing demonstration that traditional instruction does not allow most students to develop Newtonian understanding [5-7].

These conceptual instruments allow only one response per item, forcing students to choose between the correct answer and several distractors (incorrect answers), with several embodying common errors or misconceptions. Forcing the selection of a single response obscures the 
fact that many students can hold both correct and incorrect conceptions at the same time and makes it very difficult to examine the complex process of converting incorrect reasoning to correct reasoning.

In this work, we apply Bock's nominal response model (NRM) [8] from item response theory to evaluate student responses to three large samples of FCI data. The NRM models a student's probability of selecting each of the five responses to each FCI item and can extract multiple latent ability traits. We demonstrate that one ability trait is consistently associated with correct Newtonian thinking while other ability dimensions represent specific modes of incorrect thinking. Because both abilities are estimated simultaneously, the NRM allows investigation into how correct and incorrect knowledge coexist in the same student.

\section{A. The Force Concept Inventory}

The FCI is a 30-item multiple-choice instrument designed to measure a student's understanding of Newtonian mechanics [3]. The instrument was developed to probe both a correct understanding of mechanics and to present the student with responses representing common naive conceptions (misconceptions). The instrument contains items requiring a knowledge of Newton's laws, one- and two-dimensional kinematics, the law of gravitation, and a knowledge of forces. The instrument was revised from the original published instrument in 1995; this work examines the revised instrument available on PhysPort [9]. Hestenes and Jackson provided a detailed taxonomy of the misconceptions tested by the instrument in 2007 [10].

\section{B. Item response theory and the FCI}

Item response theory (IRT) represents a broad set of statistical models that predict the probability that a student will either correctly answer or will select a certain response on a multiple-choice instrument. To predict the probability, IRT estimates one or more latent traits called "abilities." If the probability of correctly answering an item is estimated, the latent ability is related to a student's overall facility with the material and is highly correlated with the instrument score. Many IRT models have been used to study the FCI including the Rasch model, the 2-parameter logistic (2PL), the three-parameter logistic (3PL), multidimensional IRT (MIRT), and the 2PL nominal response model (2PLNRM). These models are discussed in more detail in Sec. II B 1. IRT adopts the terminology of classical test theory (CTT) [11] and generally estimates a difficulty and one or more discriminations for each item. Unlike in CTT where difficulty is unintuitively related to the fraction of students who get the item correct, in the Rasch model, the 2PL model, and the 3PL model items with higher difficulty are answered correctly by fewer students. Discrimination is related to how well the item can be used to separate high and low performing students.
The Rasch model was applied to post-test data from students in Croatia in their final year of secondary education, as well as to a smaller sample of first-year university engineering students in an introductory physics course [12]. The Rasch model is a single parameter IRT model which estimates only the difficulty of each item. Items 7, 18, and 29 were not well fit by the Rasch model.

Wang and Bao used the 3PL model to examine pretest data from students in an introductory calculus-based university physics course in the United States [13]. The 3PL model estimates the difficulty and discrimination of each item and also the probability the item is answered by random guessing. They showed that the total score on the FCI was a good proxy for latent student ability estimated by IRT. All items were well fit by the 3PL model. The guessing rate was also below random chance for the majority of the items which further supported that not all of the distractors were equally attractive to students. For a five-response instrument, a student who is guessing randomly should select the correct answer $20 \%$ of the time. In 2015, the 3PL model was applied to split the FCI into two equivalent halflength exams [14]. Items 1, 3, 16, 27, and 29 were removed from the half exams due to poor psychometric functioning.

Scott and Schumayer applied MIRT, which they called multitrait IRT, to the FCI [15]. They reported that a five-dimensional model was optimal for an algebrabased introductory service course. The MIRT model did not assign items 2,3 , or 14 to a factor, leading to the suggestion that they be dropped or replaced in the instrument. Another investigation [16] applied exploratory MIRT to all 150 responses of 17000 FCI pretests; this revealed a dimension perpendicular to "Newtonian correct" that was identified as corresponding to a number of items involving impetus ideas.

In 2018, Traxler et al. used the 2PL model to study differences between the FCI scores of men and women at three institutions [17]. The 2PL model only includes a discrimination and a difficulty parameter, dropping the guessing parameter of the 3PL model. IRT found a substantial difference in difficulty by gender for items $14,21,22,23$, and 27. Item 29 was not well fit by the 2PL model.

MIRT can be used for either exploratory or confirmatory analysis. In exploratory MIRT, the model is determined entirely by the data without using a theoretical model. In confirmatory MIRT, the factor memberships are preassigned by the investigators, based on a theoretical division of the instrument. Stewart et al. [18] applied both exploratory and confirmatory MIRT to a sample of FCI data, which was also used in Traxler et al. [17] and in the present work (sample 2). Exploratory MIRT found nine factors as optimal. Much of this structure was due to the practice of blocking items. In an item block, multiple items refer to a common stem. A confirmatory analysis using a theoretical model based on a consensus of experts views of the 
principles required to answer each item correctly was then applied after the elimination of blocked items. This produced models with excellent fit, far superior to models fitted to the originally published structure of the FCI [3]. For the confirmatory model, four sets of isomorphic items requiring very similar solution structures were identified: $\{5,18\},\{17,25\},\{6,7\}$, and $\{4,15,28\}$. These isomorphic groups explained much of the factor structure not resulting from blocking. The last group represents Newton's third law; if blocked items were included, item 16 would be added to this group.

A second investigation using confirmatory MIRT was completed by Eaton and Willoughby [19] using PhysPort data [9]. Three expert models, two from previous confirmatory factor analyses and the intended structure of the creators of the FCI, were fit to pretest and post-test FCI data to measure improvement in these factors over time. None of these models fit the data well. Instead, an exploratory MIRT analysis suggested the presence of a general factor that included every item, as well as subfactors that represent individual concepts.

Polytomous IRT has been applied to understand all responses of the FCI, not just the correct responses. Eaton and Willoughby [20] applied a unidimensional 2PL nominal response model (NRM) to PhysPort data to create a partial credit model for the FCI. In a partial credit NRM, each response is related to the general correct factor to generate a score which reflects how well students who selected that response scored on the instrument as a whole. Incorrect responses chosen by students who scored higher on the exam overall are worth more partial credit. Eaton and Willoughby identified items 11, 14, 15, 16, and 29 as functioning poorly because the incorrect response chosen by those who are closest to Newtonian thinking was worth more than 0.75, which is the ideal maximum for a distractor [20].

Recently, the 2PLNRM has been used by Smith et al. to explore the FMCE and to develop a categorization of the distractors of the instrument [21]. This work reports the item characteristic curves (plots of the IRT probability functions) for multiple FMCE items. A number of the curves have maximum at intermediate IRT ability, a feature the present work will explore in the FCI.

\section{Item response curves}

Morris et al. [22] used item response curves (IRC) to examine item functioning. In an IRC analysis, students are binned by their total score and the percentage of students in each bin who selected each response is plotted. In a later study, Morris et al. found that an IRC analysis could replicate many results from IRT [23]. An IRT analysis assigns a quantitative difficulty to each item, based on what ability score is required for a $50 \%$ chance of answering correctly [24]. The percentage of students who answered an item correctly had a correlation coefficient of 0.89 with the difficulty parameters found by Wang and Bao using a 3PL model [13]. The latent ability traits extracted by IRT were highly correlated with total test score (the independent variable for IRC curves) further supporting the deep relationship of the two methods.

From the IRC analysis, a hierarchy of distractors can be created, with distractors selected more often by students with higher scores reflecting a distractor farther along the path to Newtonian thinking. A tool for classroom instructors, transition matrices, was created incorporating this hierarchy of distractors into the analysis of students' pretest to post-test transitions [25].

\section{Misconceptions}

When the FCI was created in 1992 [3], it was part of a major strand of PER research dating to the late 1970s [1,2,26-29]. In retrospect, one may describe this strand of research as the creation of a theory of instruction for introductory physics that addresses the conceptual structure that students have prior to taking a physics course [30]. These preconceptions were recognized as stable and durable ways of student thinking that were not affected by the methods of instruction in use at the time [2]. All of these preconceptions were creative and sometimes useful ways of interpreting the world; some of the preconceptions could also be used as anchoring phenomena to help students adopt Newtonian thinking with the correct form of instruction. However, in most cases, the preconceptions were impediments to learning Newtonian mechanics and incompatible with Newtonian thinking [29]. The FCI was created with the goal of becoming a diagnostic tool to identify misconceptions held by students [3].

The FCI was designed with six categories of misconceptions, based on previous research [3]. These categories, which carried through the 1995 revision of the instrument, are kinematics, impetus, active force, action-reaction pairs, concatenation of influences, and other influences on motion. A detailed taxonomy of the misconceptions measured by individual responses on the FCI was compiled by Hestenes and Jackson [10]. We will apply this taxonomy in the present work. The categories on the FCI are not mutually exclusive and some responses are in more than one category.

Some student preconceptions about kinematics are not true misconceptions, but rather that the ideas of kinematics-position, velocity, and acceleration-are not formally defined and are therefore often undistinguished $[3,28]$.

The impetus category reflects the medieval and Galilean concepts of forces commonly held by students preinstruction [1,26-28]. Impetus is the idea that a motive force is transferred to the object by the initial cause of motion. In student misconceptions, impetus can increase or decrease during motion to explain changes in the motion of the objects. A common explanation for the motion of an object 
thrown into the air, such as a flipped coin, is that impetus dissipates as the object rises, explaining why it moves upwards at first, before stopping and turning around [1]. The impetus in an object leads to the expectation that an object undergoing circular motion will continue in a circle after the centripetal force is removed [26].

Students sometimes believe that motion requires a cause, often resulting in the belief that there must be a force in the direction of motion [28]. The active forces category covers the implications of this idea, such as velocity is proportional to the applied force, therefore no force means no motion and acceleration is caused by increasing (or decreasing) forces [1].

Students' understanding of Newton's third law follows a dominance paradigm, where the more massive object or more active agent wins out and exerts more force in the direction of motion. This paradigm is not only found in the action-reaction pairs category; it also extends to the superposition of forces, where the largest force determines the motion of an object [28]. Other misconceptions in the concatenation of influences category are the idea that the motion is determined by a compromise between the forces acting on an object [28]. A final misconception is the last in-first out principle where the force that acts most recently in time determines the motion. This is seen in the problem of a spacecraft drifting laterally in space firing its aft engine [1] or using an air hose to change the path of a moving dry ice puck [27].

Finally, the other influences on motion category contains misconceptions on a variety of topics from gravity to resistive forces. The objects exert no forces misconception resides in this category. The identification of a centrifugal force during circular motion is also a part of this category. The common misconceptions surrounding gravity are in this category, including heavier objects fall faster, gravity as a quality of the mass of an object, and gravity being assisted by air pressure. The last type of misconception in this category is related to resistance. This includes the idea that objects do not begin moving until the force overcomes resistance, that the weight or mass of an object is a resistance that opposes forces, and that there is a resistive medium that produces a resistance, but not an active force [28].

The interpretation of patterns of incorrect responses to the FCI as misconceptions has recently been supported by network analytic studies showing clusters of responses are often related to identifiable misconceptions; thus showing the misconceptions are applied consistently across different physical contexts [31-33].

The misconception model of student incorrect reasoning was the predominant view through the early history of PER. As a more nuanced understanding of the complexities of student thinking was developed, alternate theories of knowledge emerged. Two important alternate theories are knowledge in pieces [34,35] and ontological categories
[36-38]. The knowledge-in-pieces theory models student thinking as composed of small fragments of reasoning that are activated to solve a problem. This model has been explored and refined by many researchers. The reasoning pieces have been described as phenomenological primitives ( $p$ prims) [34,35], facets of knowledge [39], or resources [40-42]. Unlike the misconception view, $p$ prims are not necessarily negative and may be employed to accomplish correct reasoning. The ontological categories model suggests that incorrect reasoning results from students systematically misclassifying phenomena [36-38]. For example, a student might misclassify force as a substance that could be depleted resulting in a belief that an object in motion would come to rest because of the dissipation of the provided force.

This work will identify dimensions of student reasoning beyond a correct Newtonian dimension. As a quantitative exploratory method, the analysis in this study cannot determine which theory of knowledge correctly describes these additional dimensions. It is indeed possible that there are coexisting incorrect methods of reasoning, some of which are best modeled as misconceptions, some as $p$ prims, and some as consistent categorical misclassifications.

\section{E. Conceptual change}

One the goals of PER is having students adopt a more accurate (i.e., Newtonian) conception of nature, moving the student from their misconceptions to the correct model. This process of repairing a misconception is defined as conceptual change [43]. However, this definition may be too strict, as it excludes the possibility of changing from one model to another, neither of which is Newtonian. For example, the antiperistasis theory of motion posits that thrown objects are pushed by the air, while the medieval impetus theory has an inner force that continued the motion of violently moved objects; neither theory is correct and neither is more Newtonian. A more general definition of conceptual change should then be moving from one conception to another, with the hope that the student moves to the correct model.

Subordinate to this definition is the idea of a mental model that can be modified, mollified, or replaced. A mental model is "a robust and coherent knowledge element or strongly associated set of knowledge elements" [44]. In this definition of mental models, conceptual change can happen if the knowledge elements are changed or if the associations are modified. Additionally, students may have more than one mental model that is activated by context-a student may respond with a Newtonian answer in some cases, while applying a misconception in others, with still other answers of which he or she is simply uncertain. Theories of conceptual change depend in part on the underlying source of the misconception. If it is incorrect factual beliefs, this may be repaired straightforwardly with 
correct information. On the other hand, if the association of beliefs is at issue, then there is a structural problem. Chi and Roscoe [43] describe conceptual change as a change in the categorical ontology of an idea, a substantial metacognative task. This seems to capture the idea of changing one's mental model from impetus and force in the direction of motion to instead a model of relativity of motion and Newton's laws-a substantial ontological change in what force and motion are.

Understanding conceptual change may lead to understanding how to facilitate that change. For conceptual change to happen, the student must be shown the need for such change; if they require a transformation in their conceptual ontology, a new model and ontology must be provided with the appropriate scaffolding in order to perform the metacognitive work. Direct instruction is often found to be marginally helpful in this process. Instruction that better highlights the contradictions between the old and new models or old models and observations will more likely encourage the metacognition necessary for conceptual change. Student-centered approaches appear to lead to greater conceptual change than instructor-centered methods as indicated both by measures of normalized gains as well as techniques such as model analysis $[7,44]$.

\section{F. Research questions}

This study explores the following research questions:

RQ1 Are there identifiable common morphologies of item response curves? How are the characteristics of these curves related to common student misconceptions?

RQ2 What psychometric models are suitable for modeling responses to distractors as well as the correct response?

RQ3 How can these psychometric models be used to understand the interaction of correct and incorrect knowledge?

\section{METHODS}

\section{A. Sample}

This work examines three large samples of FCI post-test responses. Sample 1 was collected from a large land-grant university in the western United States (U.S.) serving 42 000 students. Fifty percent of the undergraduate population had ACT scores in the range 22 to 29 . The demographic composition of the university was 50\% White, $21 \%$ Hispanic, $11 \%$ International, $8 \%$ Asian, 5\% two or more races, $4 \%$ Black, with other races $1 \%$ or less [45]. Sample 2 was collected from a large land-grant university in the southern U.S. serving 23000 students. Fifty percent of the undergraduate population had ACT scores in the range 23 to 29 . The demographic composition of the university was $76 \%$ White, 9\% Hispanic, 4\% Black, 4\% two or more races, $3 \%$ International, $2 \%$ Asian, with other races $1 \%$ or less [45]. Sample 3 was collected from a large land-grant university in the southern U.S. serving 18000 students. Fifty percent of the undergraduate population had ACT scores in the range 22 to 29 . The demographic composition of the university was $72 \%$ White, $19 \%$ Black, $3 \%$ Hispanic, $2 \%$ two or more races, with other races $1 \%$ or less [45]. All samples were collected over multiple years. Samples 1 and 3 were collected in many instructional environments, while the instructional environment for sample 2 was consistent through the period studied.

All samples included only post-test records without missing responses. The three samples had very different sample sizes and overall outcomes on the FCI. Sample 1 included $N=9606$ records; the average FCI post-test percentile score was $54 \pm 22$. Sample 2 included $N=4360$ records; the average FCI post-test percentage score was $71 \pm 18$. Sample 3 included $N=1439$ records; the average FCI post-test percentile score was $43 \pm 20$.

\section{B. Analysis methods}

\section{Item response theory}

Item response theory encompasses a broad collection of models. Before introducing the nominal response model applied in the current work which predicts the probability of all responses, we introduce the more intuitive 2-parameter logistic from which the NRM is generalized. The 2PL model predicts the probability $P_{s i}$ that student $s$ will correctly answer item $i$ using a logistic probability function is given by

$P_{s i}=\frac{\exp \left[a_{i}\left(\theta_{s}-b_{i}\right)\right]}{1+\exp \left[a_{i}\left(\theta_{s}-b_{i}\right)\right]}=\frac{\left.\exp \left[a_{i} \theta_{s}+d_{i}\right)\right]}{\left.1+\exp \left[a_{i} \theta_{s}+d_{i}\right)\right]}$,

where $\theta_{s}$ is the latent ability of student $s, a_{i}$ is the discrimination of item $i$, and $b_{i}$ is the difficulty of item $i$. The difficulty $b_{i}$ represents the value of $\theta_{s}$ at which a student has a $50 \%$ probability of answering the item correctly. The discrimination $a_{i}$ is related to the slope of the probability function with respect to $\theta_{s}$ at $\theta=b_{i}$. Generalizations of the 2PL model are generally written in terms of $d_{i}=-a_{i} b_{i}$. Note that this probability approaches zero as $\theta \rightarrow-\infty$ and one as $\theta \rightarrow+\infty$ if $a_{i}>0$. For well functioning items, the discrimination should be positive. The 3PL model extends the 2PL model by adding a constant term for each item representing the probability of getting the item correct by random guessing. The Rasch model constrains the discrimination to be one, $a_{i}=1$. Multidimensional IRT estimates $m$ discrimination and ability parameters for each item turning these quantities into vectors and their product into a dot product.

\section{Nominal response model}

The NRM [8] extends the 2PL model, which predicts the probability of selecting the correct response, producing a 
model which predicts the probability of selecting any of the $k=5$ responses to each item in the FCI. The probability that student $s$ selects response $j$ to item $i$ is given by Eq. (2). While the NRM can be applied with any number of ability traits (called ability dimensions), the present work primarily uses the two-dimensional model given by

$$
P_{s i j}=\frac{\exp \left[\alpha_{i j}\left(a_{i 1} \theta_{s 1}+a_{i 2} \theta_{s 2}\right)+d_{i j}\right]}{\sum_{m=1}^{k} \exp \left[\alpha_{i m}\left(a_{i 1} \theta_{s 1}+a_{i 2} \theta_{s 2}\right)+d_{i m}\right]},
$$

where $\theta_{s 1}$ and $\theta_{s 2}$ are two latent ability dimensions for student $s, a_{i 1}$ and $a_{i 2}$ are the overall discrimination of item $i$ on each ability dimension, $\alpha_{i j}$ is the discrimination of item $i$ on response $j$, and $d_{i j}$ is related to the probability of selecting response $j$ to item $i$ at $\theta_{1}=\theta_{2}=0$. The response to an item with larger $d_{i j}$ is selected by students with $\theta_{1}=$ $\theta_{2}=0$ more often than other responses to the item. While a straightforward generalization of Eq. (1), the sum in the denominator needed to normalize the probability to one serves to link all items. As such, the individual $d_{i j}, a_{i 1}$, and $a_{i 2}$ parameters cannot be interpreted independently, making the NRM model substantially less intuitive than the 2PL model. The zero and length scale of the various parameters are set by "identifying the model" which sets $d_{i 1}=0$, $\alpha_{i 1}=0$, and $\alpha_{i k}=k$.

The model has an additional degree of freedom not fixed by the identification process, the combination $a_{i 1} \theta_{s 1}+$ $a_{i 2} \theta_{s 2}$ can be extended to any number of ability dimensions by making both the ability and the discrimination into vectors; the term then becomes $\vec{a} \cdot \vec{\theta}$. The discrimination and ability only appear in $P$ as the dot product. As with any dot product, the quantity is invariant under any rotation of the coordinate system.

In contrast to the 2PL model, the NRM model can describe a probability function that has a global maximum at some finite $\theta$ which decays to zero in the limit as $\theta \rightarrow \pm \infty$. Thus, a normalized model like the NRM can produce a good fit to an item response curve with a maximum not at $\pm \infty$ [16].

The 2PL NRM model has also been used to investigate all responses in conceptual instruments [21]. The 2PLNRM models the probability of selecting any response as the product of the 2PL probability for the correct response and the NRM probability for the incorrect responses.

\section{Item response curves}

Item response curves [22] provide a useful representation of the data. An IRC curve is the fraction of students with each possible overall FCI score who answer item $i$ by selecting response $j$. Because there are often few students with some overall FCI scores, it is common to "bin" students into a range of FCI scores. An IRC curve therefore plots the effective probability of selecting each response against the FCI score. Because overall FCI score and the latent ability estimated by IRT are strongly correlated, this produces curves that are very similar to the $2 \mathrm{PL}$ item characteristic curves (ICC) for the correct response and to the NRM curves for the incorrect responses. An ICC is a plot of the probability of a response against the latent ability trait.

\section{RESULTS}

\section{A. Item response curves}

In order to investigate how the pattern of responses to the FCI change with overall Newtonian ability measured by total FCI score, IRCs were generated for every question. Figure 1 shows the IRCs for each item in the FCI post-test aggregating the three samples. To compute the IRC, students were divided into bins according to their overall score (the number of items they answered correctly); then, for each possible response, A to E, the fraction of students in each score bin was plotted. The IRC is represented by plotting a symbol showing the frequency each response is selected for each bin. Each bin contains two adjacent total scores (for example, total score 15 and 16). The total score on the FCI can take on any value from 0 to 30,31 possible scores. To divide the FCI scores into bins of width 2, students with a total score of zero were removed. Only one student in the three samples scored zero and was removed. Figure 1 also shows the ICCs (drawn as solid curves) which plot the 1D NRM probability function against the average $\theta$ of each bin. The 1D NRM is discussed in Sec. III B 1. The Supplemental Material [46] contains frequency plots of each response of each sample for readers interested in the number of students in each bin. The correct response is shown in black; note that it approaches one for every question at the highest overall score. Similar plots for each sample are provided in Ref. [46].

The curves in Fig. 1 can be classified into three distinct shapes: monotonically increasing, monotonically decreasing, and other. As one would expect, the correct response is the monotonically increasing response for all items; as FCI total score increases the fraction of students selecting each and every correct response increases. Also, as one would expect most of the incorrect response curves are monotonically decreasing; as the total score increases, the fraction of students selecting a given incorrect response decreases. There are, however, a number of responses which demonstrate a different morphology, either having an extended horizontal region or initially increasing before peaking and decreasing. For these curves, increasing overall performance on the FCI either does not change the likelihood of selecting the incorrect response or increases the likelihood (at lower total scores) of selecting the incorrect response. These anomalous curves are called "intermediate maximum" (IM) curves in this work. Such IM curves are even more prevalent in analyses of pretest results [16]. 

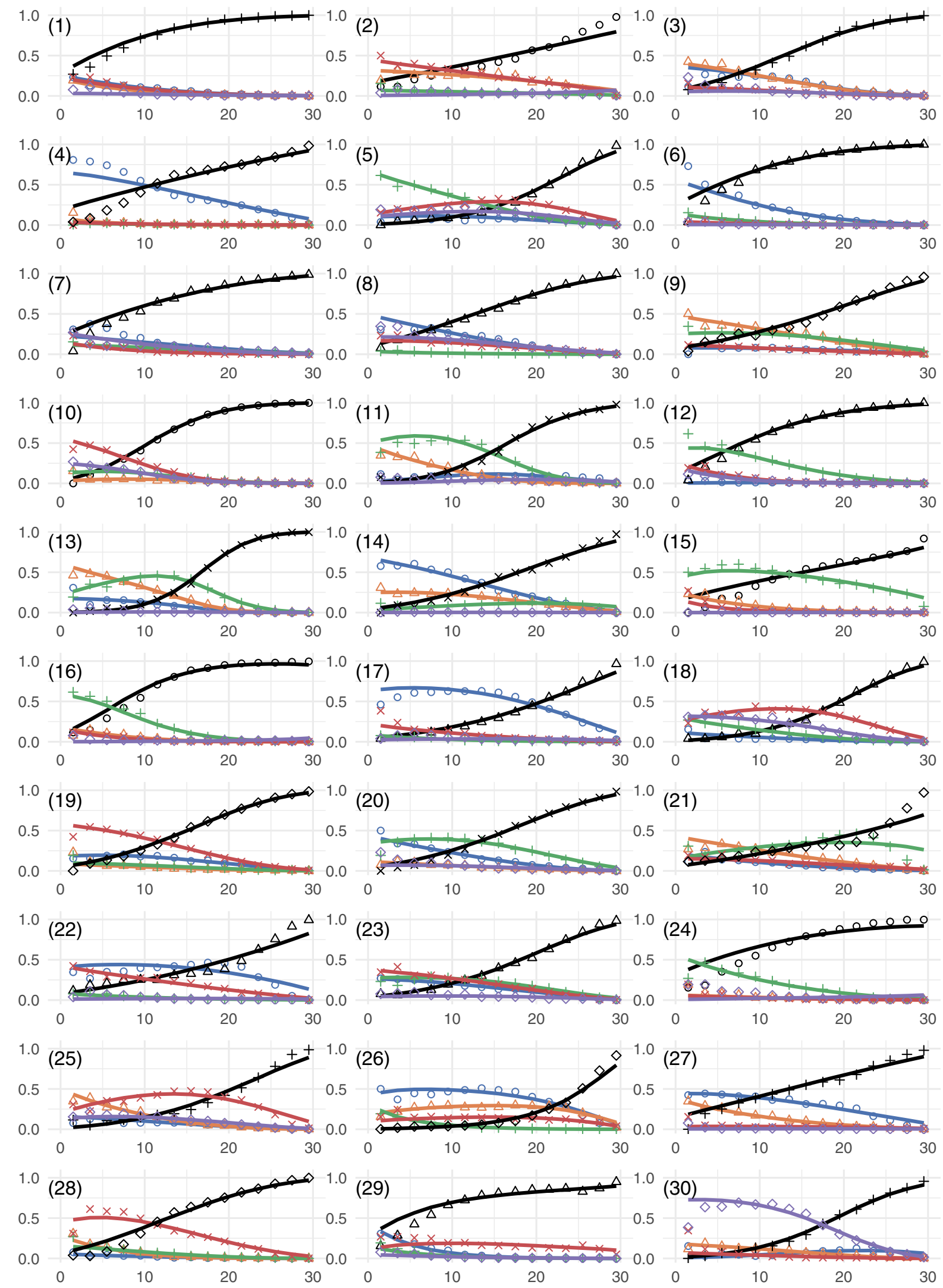

FIG. 1. Item response curves for all FCI items in the aggregated dataset plotted as individual symbols. For incorrect responses, (A) is blue (circle), (B) is orange (triangle), (C) is green (plus), (D) is red (cross), and (E) is purple (diamond). Item characteristic curves for the 1D NRM for all FCI items in the aggregated dataset are drawn as solid curves. 
TABLE I. Intermediate maximum IRC curves. Responses labeled with a superscript $f$ are flat over an extended range, other items reach a maximum before decaying.

\begin{tabular}{|c|c|c|c|c|c|}
\hline FCI item & Combined & Sample 1 & Sample 2 & Sample 3 & Misconception \\
\hline 2 & $\mathrm{~B}^{f}$ & $\mathrm{~B}$ & & & Heavier objects fall faster \\
\hline 3 & $\mathrm{~A}^{f}$ & $\mathrm{~A}^{f}$ & & & $\begin{array}{l}\text { Force causes acceleration to terminal velocity } \\
\text { Impetus supplied by "hit" }\end{array}$ \\
\hline 5 & $\mathrm{D}$ & $\mathrm{D}$ & $\mathrm{D}^{f}$ & $\mathrm{D}$ & $\begin{array}{l}\text { Circular impetus } \\
\text { Motion implies active force }\end{array}$ \\
\hline 9 & $C^{f}$ & $\mathrm{C}^{f}$ & & $\mathrm{C}$ & Nonvectorial velocity composition \\
\hline 11 & $\mathrm{C}$ & $\mathrm{C}$ & & $\mathrm{C}^{f}$ & Impetus supplied by "hit" \\
\hline 13 & $\mathrm{C}$ & $\mathrm{C}$ & & $\mathrm{C}$ & Impetus dissipation \\
\hline 14 & & & $\mathrm{C}$ & & Force compromise determines motion \\
\hline 15 & & & & $\mathrm{C}^{f}$ & Most active agent produces greatest force \\
\hline 17 & A & A & $\mathrm{A}^{f}$ & $\mathrm{~A}^{f}$ & Largest force determines motion \\
\hline 18 & $\mathrm{D}$ & $\mathrm{D}$ & $\mathrm{D}$ & $\begin{array}{c}\mathrm{D} \\
\mathrm{E}^{f}\end{array}$ & $\begin{array}{l}\text { Circular impetus } \\
\text { Centrifugal force }\end{array}$ \\
\hline 20 & $\mathrm{C}^{f}$ & $\mathrm{C}^{f}$ & & $\mathrm{C}^{f}$ & Velocity-acceleration undiscriminated \\
\hline 21 & $\mathrm{C}$ & $\mathrm{C}$ & $\mathrm{C}$ & $\mathrm{C}$ & Force compromise determines motion \\
\hline 22 & A & A & A & A & Velocity proportional to applied force \\
\hline 23 & $\mathrm{C}$ & $\mathrm{C}$ & & & Last force to act determines motion \\
\hline 25 & $\mathrm{D}^{f}$ & $\mathrm{D}$ & $\mathrm{D}^{f}$ & $\mathrm{D}$ & Motion when force overcomes resistance \\
\hline & A & A & & $\mathrm{A}^{f}$ & Velocity proportional to applied force \\
\hline 26 & & $\mathrm{~B}^{f}$ & $\mathrm{~B}$ & $\mathrm{~B}^{f}$ & $\begin{array}{l}\text { Motion when force overcomes resistance } \\
\text { Resistance opposes force or impetus }\end{array}$ \\
\hline 30 & $\mathrm{E}^{f}$ & $\mathrm{E}^{f}$ & & $\mathrm{E}$ & Impetus supplied by "hit” \\
\hline
\end{tabular}

Table I presents a summary of the IM curves found in each sample and in the aggregate (combined) sample. This classification was performed using the IRC represented as individual symbols, not a solid curve, in Fig. 1. Each curve is labeled with the misconception tested by the response as identified in Hestenes and Jackson's taxonomy [10]. We denote the B response to FCI item 1 as "1B." While some IM curves are only found in one sample (2B, 3A, 14C, 15C, $18 \mathrm{E}, 20 \mathrm{C}$, and $23 \mathrm{C}$ ), many are found in two samples (9C, $11 \mathrm{C}, 13 \mathrm{C}, 20 \mathrm{C}, 26 \mathrm{~A}, 26 \mathrm{~B}$, and 26E) or in all three samples $(5 \mathrm{D}, 17 \mathrm{~A}, 18 \mathrm{D}, 21 \mathrm{C}, 22 \mathrm{~A}$, and $25 \mathrm{D})$. This last group is particularly interesting because is contains pairs of items identified as measuring consistently applied incorrect reasoning, possibly representing strongly held misconceptions, in recent network analyses of the FCI $[32,33]$. Item pairs $\{5,18\}$ and $\{17,25\}$ were also identified as requiring similar reasoning for their correct solution in a constrained MIRT analysis [18].

For the following we utilize the modifications to the Hestenes and Jackson taxonomy [10] suggested by the network analytic studies of Wells et al. [32] which explain the commonalities observed more thoroughly. Responses 5D and 18D are consistently selected by students applying the motion implies active forces misconception [32]. Responses 17A and 25D are consistently selected by students applying largest force determines motion misconception. Responses $21 \mathrm{C}$ and $22 \mathrm{~A}$ are part of a 4-item block referring to a common stem. The practice of blocking items has been shown to generate spurious correlations between the items which may explain why both have similar structure, in this case pronounced IM structure. Responses $11 \mathrm{C}$ and $13 \mathrm{C}$ were also consistently selected by students applying the motion implies active forces misconception. One set of consistently applied misconceptions identified in both the FCI [32] and the FMCE [47] is absent; the Newton's third law misconceptions of greater mass implies greater force and most active agent produces greatest force measured by $\mathrm{FCI}$ responses 4A, 15C, 28D. FCI item 16 also involves Newton's third law but is not consistently associated with the other Newton's third law items in network analysis or factor analysis.

\section{B. Item response theory}

To quantitatively explore the IM structure and to develop an understanding of the state of student understanding implied by the structure, multiple IRT models were investigated.

\section{One-dimensional models}

To establish a baseline, the 2PL model was fit to the dichotomously scored items; this analysis scores each item as correct or incorrect. The results are presented in the first row of Table II. Multiple model fit statistics are reported. $\mathrm{Hu}$ and Bentler recommend using multiple fit statistics to characterize a model [48]. We report the Bayesian 
TABLE II. Model fit statistics for individual samples.

\begin{tabular}{|c|c|c|c|c|c|c|c|c|c|c|c|c|}
\hline \multirow[b]{2}{*}{ Model } & \multicolumn{4}{|c|}{ Sample 1} & \multicolumn{4}{|c|}{ Sample 2} & \multicolumn{4}{|c|}{ Sample 3} \\
\hline & BIC & RMSEA & TLI & CFI & BIC & RMSEA & TLI & CFI & BIC & RMSEA & TLI & $\mathrm{CFI}$ \\
\hline 2PL & 319513 & 0.060 & 0.921 & 0.926 & 122940 & 0.071 & 0.837 & 0.849 & 48072 & 0.056 & 0.909 & 0.915 \\
\hline 1D NRM & 591501 & 0.042 & 0.892 & 0.929 & 187551 & 0.055 & 0.776 & 0.854 & 99773 & 0.041 & 0.828 & 0.888 \\
\hline 2D NRM & 586346 & 0.028 & 0.952 & 0.973 & 184168 & 0.028 & 0.942 & 0.967 & 99298 & 0.030 & 0.907 & 0.947 \\
\hline 3D NRM & 583887 & 0.025 & 0.962 & 0.982 & 183504 & 0.021 & 0.966 & 0.983 & 99323 & 0.026 & 0.931 & 0.966 \\
\hline 4D NRM & 583110 & 0.026 & 0.960 & 0.984 & 182833 & 0.022 & 0.964 & 0.985 & 99322 & 0.027 & 0.928 & 0.970 \\
\hline 5D NRM & 582413 & 0.021 & 0.974 & 0.991 & 182224 & 0.018 & 0.977 & 0.992 & 99395 & 0.023 & 0.948 & 0.983 \\
\hline 6D NRM & 581531 & 0.019 & 0.979 & 0.995 & 182107 & 0.019 & 0.974 & 0.993 & 99419 & 0.024 & 0.941 & 0.985 \\
\hline 7D NRM & 581475 & 0.022 & 0.972 & 0.995 & 182097 & 0.016 & 0.980 & 0.996 & 99545 & 0.020 & 0.959 & 0.992 \\
\hline 8D NRM & 581194 & 0.018 & 0.981 & 0.998 & 181819 & 0.019 & 0.974 & 0.997 & 99745 & 0.024 & 0.941 & 0.993 \\
\hline 9D NRM & 581407 & 0.026 & 0.959 & 0.998 & 181907 & 0.010 & 0.993 & 1.000 & 99825 & 0.029 & 0.917 & 0.995 \\
\hline
\end{tabular}

information criterion (BIC), the root mean square error of approximation (RMSEA), the Tucker-Lewis index (TLI), and the comparative fit index (CFI). Optimal models minimize BIC with changes in BIC of 20 considered "very strong" [49]. Hu and Bentler suggest RMSEA $<0.05$, TLI $>0.96$, and CFI $>0.96$ for acceptable model fit [48]. As shown in Table II, the 2PL model does not meet the criteria for acceptable model fit. A lack of fit is also evident in prior studies of the FCI; the ICCs reported by Wang and Bao [13] often have poor fit in the tails of the curve.

Polytomous models, which fit the correct response as well as the distractors, were then applied. It is not possible to fit the maximum in the IM curves using a model such as the 2PL model that is monotonically increasing (or decreasing for negative discrimination). The NRM provides a generalization of the $2 \mathrm{PL}$ model by normalizing the sum of predicted probabilities for all responses to an item to unity as shown in Eq. (2). The NRM, like the 2PL model, also naturally generalizes to multiple latent ability dimensions. A substantial number of studies have suggested from 5 to 9 factors are needed to describe the correct answer structure of the FCI $[15,18,50,51]$; as such, the combined correct and incorrect answer structure is expected to be multidimensional. In Table II, using one latent ability trait, the one-dimensional nominal response model (1D NRM) produced generally superior RMSEA but weaker TLI and CFI than the 2PL model. The 1D NRM model therefore does not meet the criteria of acceptable fit. The 1D NRM model is the IRT model most closely related to the IRC of the previous section. The 1D NRM ICCs (shown as solid curves) were plotted for each FCI item in the aggregated dataset in Fig. 1 by calculating an average $\theta$ for each bin; the plots for the individual samples are presented in the Supplemental Material [46]. While not meeting the criteria of acceptable fit, in general, the ICC are a very good visual fit to the data for most items in all samples. We note the visual fit is somewhat extraordinary considering five curves are fit for each item using a single student-level latent ability for all items.
The 1D NRM is the model most related to the IRCs in Fig. 1 and can be used to understand the origin of the IM curves. The latent ability $\theta$ of the 1D NRM is highly correlated to the latent ability in the 2PL model $(r=0.99)$ where $r$ is the correlation coefficient. The agreement is extraordinary because the correct answers are not identified in any way in the 1D NRM (as they are in the 2PL).

Intermediate maximum curves have two defining properties: (i) they are responses selected by a substantial number of students with moderate overall FCI score and (ii) they have a slope of zero generally near the average FCI score, which would correspond to $\theta=0$ in the 1D NRM if the overall distribution is fairly symmetric. The derivative of the probability of selecting response $j$ of item $i$ with respect the ability $\theta_{\ell}$ is shown in Eq. (3) for the general multidimensional case. The student subscript $s$ has been suppressed to simplify the following expression:

$$
\frac{\partial P_{i j}(\vec{\theta})}{\partial \theta_{\ell}}=a_{i \ell} P_{i j}(\vec{\theta})\left(\alpha_{i j}-\sum_{m=1}^{k} \alpha_{i m} P_{m}(\vec{\theta})\right) .
$$

For Eq. (3) to be zero at $\theta_{\ell}=0$, the quantity in parenthesis must be zero. At $\vec{\theta}=0$, the probability, $P_{i j}$, depends only on $d_{i j}$. If only two responses are selected at high frequency, the correct response (CR) and one incorrect response, the incorrect response must be monotonically decreasing, because the sum of the two probabilities must be one and the correct response is monotonically increasing. As such, a minimum of three responses selected by substantial numbers of students is needed to have an IM response. These will generally be an incorrect response, the correct response, and the intermediate maximum response. To understand the conditions which may produce an IM response, assume all three responses are selected at the same rate at $\theta=0$ which implies $P_{\mathrm{CR}}(0) \approx P_{\mathrm{IM}}(0) \approx$ $P_{\mathrm{IR}}(0) \approx \frac{1}{3}$. With this assumption, the condition for zero slope becomes $\alpha_{\mathrm{IM}}-\frac{1}{3}\left(\alpha_{\mathrm{CR}}+\alpha_{\mathrm{IM}}+\alpha_{\mathrm{IR}}\right)=0$ or $\alpha_{\mathrm{IM}}=\frac{1}{2}\left(\alpha_{\mathrm{CR}}+\alpha_{\mathrm{IR}}\right)$. Hence, the discrimination of the IM 
curve must be between the discriminations of the correct and the incorrect curves.

\section{Higher dimensional models}

The lack of acceptable model fit of both the 2PL and the 1D NRM models could have been predicted from the numerous research studies showing the factor structure of the instrument contains from 5 to 9 dimensions. Adding a second latent ability trait to form the 2D NRM greatly improved model fit (Table II). For samples 1 and 2, both RMSEA and CFI were in the range of acceptable fit, while TLI was near this range. Fit statistics were generally weaker for the lower performing and smaller sample 3; only RMSEA was in the range of acceptable fit for this sample. The 2D NRM is of particular interest as will be discussed in Sec. III B 3. While it is an acceptable fitting model, it is not the optimal model.

Higher dimensional models were also fit. For sample 1, all fit statistics suggest the 8D NRM as optimal. For sample 2, BIC also suggest the 8D NRM as optimal; however, other statistics support the 9D NRM. For sample 3, model selection is more complicated; BIC suggests the 2D NRM as optimal; RMSEA and TLI suggests the 7D NRM, and CFI suggests the 9D NRM.

\section{Rotations and the 2D NRM}

The dot product $\vec{a} \cdot \vec{\theta}$ in the NRM is invariant under rotations. If $\vec{a}$ and $\vec{\theta}$ are rotated through the same angle, the probabilities predicted by the model do not change. To eliminate this degeneracy, the 2D NRM model was rotated so that the $\theta_{1}$ ability dimension was maximally correlated with the $2 \mathrm{PL}$ ability trait. In all cases, the rotated $\theta_{1}$ for the 2D NRM model was very highly correlated with the ability in the 2PL model (sample 1: $r=0.99$, sample 2: $r=0.99$, sample 3: $r=0.98$ ). The high correlation shows that under this rotation, $\theta_{1}$ is related to the ability of the student to answer FCI items correctly.

Developing an intuitive understanding of the 2D NRM is challenging because the answering probabilities are coupled through the sum in the denominator. One method of understanding the meaning of the two ability dimensions is to use the two dimensional generalization of Eq. (3) to determine the derivative of the probability of selecting a response with respect to each latent trait for students of average ability $(\vec{\theta}=0)$. For all samples, the responses with the largest positive slopes with respect to $\theta_{1}$ at $\theta_{1}=0$ were the correct responses to each item. We note, again, that at no point were the correct responses defined for the algorithm; they were identified organically by the algorithm. This further supports the association of $\theta_{1}$ with correct Newtonian thinking; at average values of $\theta_{1}$ the probability of answering correctly is rapidly increasing with $\theta_{1}$ for all items.

For each sample, the Supplemental Material [46] presents a table of the slope of the probability of selecting each response with respect to $\theta_{1}$ and $\theta_{2}$. The items with largest positive slope were fairly different for each sample.

For sample 1, the largest slopes (above 0.30), shown in parenthesis, were $13 \mathrm{D} *(0.57), 11 \mathrm{D} *(0.43), 18 \mathrm{~B} *(0.41)$, $28 \mathrm{E} *(0.40), \quad 20 \mathrm{D} *(0.39), \quad 30 \mathrm{C} *(0.37), \quad 14 \mathrm{D} *(0.32)$, $5 \mathrm{~B} *(0.31), 15 \mathrm{~A}^{*}(0.31)$, and $23 \mathrm{~B} *(0.31)$. Responses marked with "**" are the correct response. These items cover a broad variety of topics from 2D kinematics, to circular motion, to Newton's third law. For sample 2, the largest positive slopes were $25 \mathrm{C}^{*}(0.40), 18 \mathrm{~B} *(0.35), 17 \mathrm{~B} *(0.33)$, and $22 \mathrm{~B} *(0.32)$. While this list does not contain the Newton's third law items, the slopes of items $4 E^{*}(0.27)$, $15 \mathrm{~A} *(0.26)$, and $28 \mathrm{E} *(0.27)$ were nearly as large. Again, this list contains items testing a broad range of Newtonian skills. For Sample 3, the largest slopes were $28 \mathrm{E}^{*}(0.53)$, $10 \mathrm{~A} *(0.37), 23 \mathrm{~B} *(0.37)$, and $13 \mathrm{D} *(0.35)$. The Newton's third law items again generally had a substantial slope: $4 \mathrm{E}^{*}(0.29)$ and $15 \mathrm{~A}^{*}(0.17)$. Examining the responses above, there does not seem to be particular topics unique to these large slope responses; larger values of $\theta_{1}$ seem to be generally associated with answering Newtonian mechanics questions correctly.

For most items, there are also responses with a substantial negative slope. For these responses, larger $\theta_{1}$ makes the probability of selecting the correct response less likely, or, conversely, smaller values of $\theta_{1}$ makes selecting the incorrect response more likely; therefore, smaller values of $\theta_{1}$ are associated with selecting incorrect responses to FCI items. For most items with a correct response with a large positive slope, there was also a single response with a commensurately large negative slope. For a few items, there were two incorrect responses with large negative slopes.

There were far fewer slopes with respect to $\theta_{2}$ that were substantially positive. For sample 1, by far the largest positive slopes were for items $4 \mathrm{~A}(0.27), 15 \mathrm{C}(0.38)$, and $28 \mathrm{D}(0.18)$. These items all test Newton's third law and these responses have been previously identified as representing consistently applied misconceptions [32,33]. For sample 2, response $15 \mathrm{C}(0.43)$ had the largest slope, followed by $22 \mathrm{~B} *(0.32), 21 \mathrm{E}^{*}(0.28), 4 \mathrm{~A}(0.21)$, $27 \mathrm{C} *(0.14), 23 \mathrm{~B} *(0.13)$, and $26 \mathrm{E} *(0.12)$. For sample 2 , increasing $\theta_{2}$ was associated with incorrectly answering Newton's third law as well as correctly answering some kinematic items.

The groups of kinematic items were all part of item blocks and it is unclear if the blocking of items partially responsible for the mixing of correct and incorrect items in $\theta_{2}$ for this sample. The $\theta_{1}$ slopes for these items were generally commensurate with the $\theta_{2}$ slopes: $21 \mathrm{E}^{*}(0.27)$, $22 \mathrm{~B} *(0.32)$, and $27 \mathrm{C} *(0.18)$. In sample 3 , the second dimension's largest slopes were the three Newton's third law misconceptions, $4 \mathrm{~A}(0.45), 15 \mathrm{C}(0.36)$, and $28 \mathrm{D}(0.20)$ as well. As such, the second ability dimension is consistently associated with the a response representing a strong misconception. 


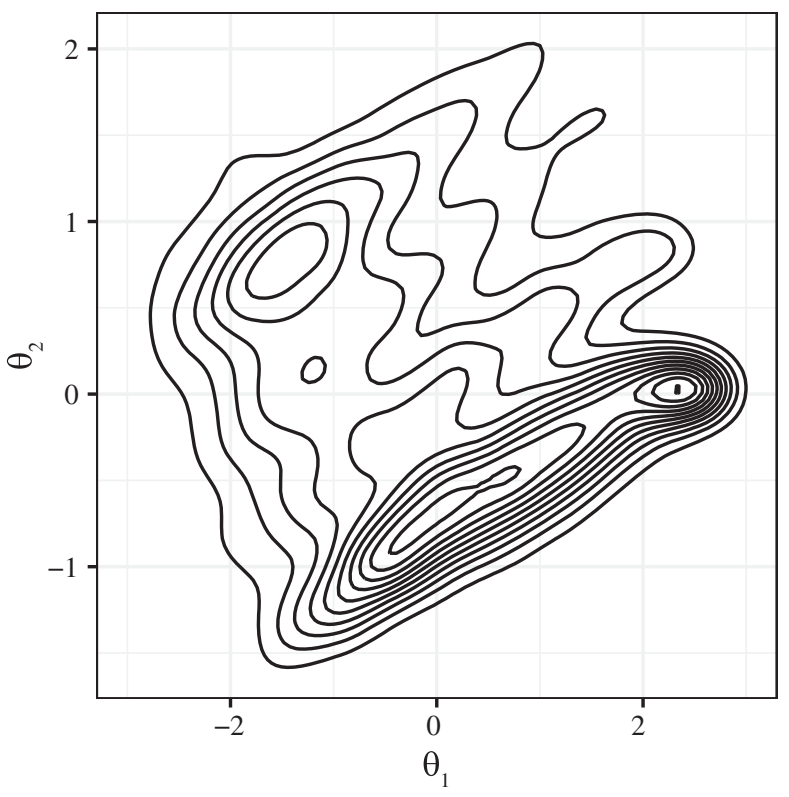

FIG. 2. Density contours plotting $\theta_{2}$ vs $\theta_{1}$ for sample 1 .

As was the case for $\theta_{1}$, there were generally responses with large negative slopes on the items with responses with large positive slopes. The items with large negative slopes were the correct response for all incorrect responses with large positive slopes. As such, while larger values of $\theta_{2}$ are associated with a tendency to answer Newton's third law items incorrectly, negative values of $\theta_{2}$ are associated with a tendency to answer Newton's third law items correctly.

\section{The clustering of ability scores}

The rotated 2D NRM allows the examination of a student's ability to answer FCI items correctly $\left(\theta_{1}\right)$ and their propensity to apply common misconceptions $\left(\theta_{2}\right)$ simultaneously. While theories of conceptual change have posited the need to replace misconceptions with correct knowledge implying both correct knowledge and the misconceptions exist in student thinking simultaneously, it has been challenging to investigate the relative role of incorrect and correct reasoning in student thinking quantitatively. To develop a picture of the patterns of incorrect and correct reasoning ability, cluster analysis was applied to the three samples. Figure 2 shows the ability density contours for sample 1. Similar figures for samples 2 and 3 are shown in the Supplemental Material [46]. All three samples show three distinct groupings of student ability. The dendrogram and skree plots are also presented in the Supplemental Material [46]. For samples 1 and 2, the dendrogram strongly supports a three-cluster solution, for sample 3 a two-cluster solution is more strongly supported although there is evidence of a three-cluster solution. In all samples, there is also evidence of a five-cluster solution. Because the density plots also provide evidence for three clusters, the three-cluster model was further investigated. Note that $\theta_{i}$
TABLE III. Cluster centers for each sample.

\begin{tabular}{|c|c|c|c|}
\hline \multicolumn{4}{|c|}{ Sample 1} \\
\hline Cluster & $N$ & $\theta_{1}$ & $\theta_{2}$ \\
\hline 1 & 3255 & 1.37 & 0.19 \\
\hline 2 & 3200 & -0.38 & -0.62 \\
\hline 3 & 3151 & -1.28 & 0.62 \\
\hline \multicolumn{4}{|c|}{ Sample 2} \\
\hline 1 & 1729 & 0.99 & 0.36 \\
\hline 2 & 1421 & -0.36 & -0.81 \\
\hline 3 & 1210 & -1.49 & 0.65 \\
\hline \multicolumn{4}{|c|}{ Sample 3} \\
\hline 1 & 421 & 1.56 & 0.20 \\
\hline 2 & 422 & -0.38 & -0.88 \\
\hline 3 & 596 & -0.96 & 0.46 \\
\hline
\end{tabular}

are normalized to have mean 0 and standard deviation 1 for each sample.

The cluster centroids, the average value of the ability traits of students in each cluster, are presented in Table III. While the three samples have substantially different average FCI post-test score, the centroids are strikingly similar. The first cluster in all samples represents students with high Newtonian ability, $\theta_{1}$, and average non-Newton's third law ability, $\theta_{2}$. Even though this cluster has students with the most correct Newtonian views, they still harbor misconceptions. Higher values of $\theta_{2}$ are associated with the selection of common misconceptions about Newton's third law. The second cluster represents students with below average Newtonian ability and well below average nonNewton's third law ability. These students' scores on the instrument are generally the result of a lack of correct knowledge, not strongly held misconceptions. The third cluster represents students with very low Newtonian ability and above average non-Newton's third law ability; these students are hampered by both a lack of correct knowledge and strongly held misconceptions. Examination of the number of students in each cluster shows that the differences in overall performance of the three samples can be explained by the relative occupation of the three clusters. In highly performing sample 2 , the majority of the students are in Newtonian cluster 1. In sample 1, students are approximately evenly split between the three clusters. In the lowest performing sample, sample 3, more students are in cluster 3 which both has low average Newtonian ability and above average non-Newton's third law ability.

\section{DISCUSSION}

This study sought to answer three research questions; they will be addressed in the order proposed.

RQ1: Are there identifiable common morphologies of item response curves? How are the characteristics of these curves related to common student misconceptions? This study identified three general shapes of item response 
curves: monotonically increasing, monotonically decreasing, and intermediate maximum. Intermediate maximum curves reach a maximum, usually between a total score of 8 and 20 (the maximum possible on the FCI is 30). The monotonically increasing curves were uniformly associated with the correct response, the monotonically decreasing curves with incorrect responses. All IM curves were associated with incorrect responses with the surprising feature that at low student overall FCI score, the probability of selecting the incorrect response increases with increasing overall FCI score. Table I shows that each IM curve was associated with a misconception in Hestenes and Jackson's taxonomy of the misconceptions measured by the instrument [10]; however, many of the responses associated with misconceptions do not have IM curves.

The association of IM curves with known and often strongly held misconceptions suggests the following explanation for IM curves. Students with very low FCI scores typically have a very disorganized knowledge of mechanics and many mistaken beliefs. Most of the well known widely held misconceptions represent a coherent, if non-Newtonian, model that correctly explains some but not all phenomena of mechanics. These may enable that student to outperform a student using less well formed models on the FCI. As such, a student applying some, even incorrect, coherent thinking on the exam might outperform students with no coherent model at lower FCI total scores.

Recent network analytic work on the FCI provides support that IM responses represent coherent thinking [32,33]. Modified module analysis (MMA) identifies collections of incorrect responses which are preferentially answered together and represent coherently applied incorrect thinking. The groups of items $\{5 \mathrm{D}, 18 \mathrm{D}\}$, $\{11 \mathrm{C}, 13 \mathrm{C}, 30 \mathrm{E}\},\{17 \mathrm{~A}, 25 \mathrm{D}\}$, and $\{21 \mathrm{C}, 22 \mathrm{~A}\}$ were identified as incorrect answering communities. These represent 9 of the 19 IM curves identified. This, however, cannot be the full explanation of the origin of IM curve because some communities representing common misconceptions are conspicuously missing from the IM curves. In particular Newton's third law $\{4 \mathrm{~A}, 15 \mathrm{C}, 28 \mathrm{D}\}$ and circular impetus $\{6 \mathrm{~A}, 7 \mathrm{~A}\}$ IRC's might be called "dominant wrong" because they increase monotonically as score decreases and are the most common responses of the lowest scoring students, attracting at least 40 percent of responses of those students. The attractiveness of the Newton's third law misconceptions, $\{4 \mathrm{~A}, 15 \mathrm{C}, 28 \mathrm{D}\}$, is particularly pronounced. These responses are selected by over $50 \%$ of the lowest performing students, showing that these misconceptions appeal strongly to the least Newtonian thinkers.

RQ2 What psychometric models are suitable for modeling responses to distractors as well as the correct response? To move beyond dichotomous grading, which primarily measures the degree of Newtonian thinking, the NRM was used to model all responses to the FCI. Analysis of all responses will further understanding of the relation between selecting the correct response to some items and incorrect responses to other items. The previous research question identified IM structure as an unexpected feature of some incorrect response curves. To model IM structure requires an extension of 2PL IRT which allows the curves to reach a maximum at finite ability values.

Smith et al. applied the 2PL NRM to all responses to the FMCE in order to establish a hierarchy of incorrect responses [21]. The 2PL NRM models the probability of a student selecting a response as the product of the $2 \mathrm{PL}$ model for the correct response and the NRM model for the four incorrect responses [52]. The 2PL NRM explicitly treats the correct and incorrect answer asymmetrically. One goal of the current study was to explore the coexistence of correct and incorrect knowledge; to do this we wanted to treat correct and incorrect thinking as of equal importance. As such, we chose the NRM which does not identify the correct answer.

After rotation, one of the ability dimensions of the 2D NRM was very strongly correlated $(r>0.98)$ with the 2PL correct latent ability in all samples. The second ability dimension was most related to strongly held Newton's third law misconceptions. While the fit statistics for the 2D NRM for samples 1 and 2 were acceptable based on $\mathrm{Hu}$ and Bentler's criteria [48], the smaller lower performing sample 3 was not as well fit. All three samples were better fit by higher dimensional models. This was to be expected from multiple factor analytic studies of the FCI suggesting the instrument is well represented by from 5 to 9 factors $[15,18,50,51]$. If the correct responses require 5 to 9 factors, the correct and incorrect answers together should require more than 5 to 9 factors.

RQ3 How can these psychometric models be used to understand the interaction of correct and incorrect knowledge? Multidimensional NRM models allow the extraction of multiple latent ability traits representing correct and incorrect thinking. These ability dimensions have many applications in the exploration of the relation of correct and incorrect knowledge. This work focused on the 2D NRM model. Cluster analysis extracted three clusters for each sample. For samples 1 and 2, the three-cluster solution was strongly suggested by the dendrogram; for sample 3 a two cluster solution was suggested with some support for a three-cluster solution. The clusters extracted were strikingly similar even though the overall FCI scores of the three samples were quite different. This suggests instructors may be able to view their classes as containing three general types of students postinstruction. The first has strong Newtonian knowledge $\left(\theta_{1}\right.$ in Table III), but still average non-Newton's third law thinking. This suggests, that even for students with a strong ability to answer conceptual mechanics problems correctly, substantial misconceptions remain and compete with the correct knowledge. The second type of student has below average Newtonian 
ability and well below average non-Newton's third law ability. For these students, the failure to apply Newton's laws correctly is largely the result of incorrect understanding of those laws and not the result of applying compelling alternate models. The third group of students has very low Newtonian ability and relatively high nonNewton's third law ability. The failure of these students to correctly answer mechanics questions results from both poor Newtonian knowledge and the application of incorrect beliefs. Each group requires different pedagogical techniques to move students in the group to a state of high Newtonian ability and low non-Newton's third law ability, to accomplish conceptual change.

\section{IMPLICATIONS AND FUTURE DIRECTIONS}

The NRM allowed the development of statistical models of the response patterns to all responses in the FCI and the extraction of multiple ability dimensions. This offers the possibility of explicitly studying the implications of different distractors, hopefully giving insight into specific details of incorrect thinking (misconceptions). The present work could only begin the process of understanding the complex relation of these ability dimensions and how they evolve with different instruction.

The similarity of the cluster centroids in Table III despite the large difference in overall FCI score at the three institutions suggests that the existence of these three subgroups of students may be general. The robustness of the clusters might allow instructors to classify their students by the relative populations of the three groups of students on a pretest and to plan instructional modifications based on the relative populations of these groups.

The co-existence of IRCs with intermediate maximum structure as well as with monotonic decreasing structure indicates that the most probable incorrect responses of students varies with overall score. This provides instructors a relatively simple analysis method to identify which misconceptions are most important in their classes. Because these IM responses are answered with higher frequency by students with moderate ability than other incorrect responses, there may be some temptation to categorize these responses as less incorrect than other incorrect responses and view transitions from non-IM to IM incorrect responses as a positive result. This seems unwise or at least requires more research to justify it. Indeed, if IM responses do generally represent strongly held misconceptions, students with these responses may be harder to transition to Newtonian thinking than students with other non-IM responses and therefore they may represent a bigger challenge for instructors to address.

This work explored the NRM in two dimensions; higher dimensional models should be examined in the future. Such higher dimensional models will require the development of a rotation algorithm in higher dimensions.
Another interesting direction is to study the effects of instruction in moving students in the various dimensions between pretests and post-tests. Contrasting the finding of Perez-Lemonche et al. [16] that impetuslike views dominate the second dimension on the FCI pretest with our finding that Newton's third law dominates this dimension on the post-test illustrates the potential for such analysis to reveal systematic shifts in the misconceptions associated with instruction. This would open the way for learning how to remediate specific misconceptions identified by multidimensional analyses.

\section{CONCLUSION}

An item response curve analysis identified three general shapes of response curves for three large FCI samples: monotonically increasing, monotonically decreasing, and intermediate maximum. These curves were plotted against total FCI score. As one would expect, the monotonically increasing curves all represented the correct response (as total score increases, the probability of selecting an individual correct response increases); the monotonically decreasing curves all represented incorrect responses. The IM curves also all represented incorrect responses, but the probability of selecting these incorrect responses unexpectedly increased with total FCI score for scores below the maximum. All of the IM responses matched common misconceptions as identified by Jackson and Hestenes [10]; however, two of the most consistently applied misconceptions involving Newton's third law-greater mass and greater activity both imply greater force-did not have IM response curves.

To allow the exploration of all responses and to further understand IM responses, the NRM was used to model all response curves of the FCI, including both correct and incorrect curves. Without identifying the correct responses within the model, the 1D NRM ability was highly correlated $(r=0.99)$ with the ability from the 2PL IRT model using dichotomously scored items. The NRM also allows the construction of response curves that reach a maximum at a finite ability.

Higher dimensional NRM models were also fit. Multiple fit statistics showed that the NRM fits significantly better with more dimensions, typically 5-8 were optimal for samples from three different large universities. These higher dimensional models have a rotational invariance which requires the selection of rotation to interpret the model, just as many factor models apply a rotation.

The 2D NRM was rotated so one dimension was maximally correlated with the $2 \mathrm{PL}$ ability to select the correct answer. This produced one dimension that was very highly correlated with the 2PL ability $(r>0.98)$. The second dimension was then most related to the misapplication of Newton's third law.

Cluster analysis was then used to understand the general distribution of the two ability dimensions at the three 
institutions. All three samples showed three main clusters: one had high Newtonian ability and neutral incorrect ability, the second slightly below average Newtonian and incorrect ability, the third had well below average Newtonian ability and above average incorrect ability. The clusters centers were remarkably similar for the three institutions; the large differences in overall FCI score between the institutions could be explained by the different relative populations of the clusters. The existence of these possibly universal clusters of students suggest multiple populations of non-Newtonian thinkers remain post instruction which may each require specific pedagogical innovations to become Newtonian thinkers.

\section{ACKNOWLEDGMENTS}

This work was supported in part by the National Science Foundation as part of the evaluation of improved learning for the Physics Teacher Education Coalition, PHY-0108787. We are grateful for contributions from Massachusetts Institute of Technology as well.
[1] J. Clement, Students' preconceptions in introductory mechanics, Am. J. Phys. 50, 66 (1982).

[2] I. A. Halloun and D. Hestenes, The initial knowledge state of college physics students, Am. J. Phys. 53, 1043 (1985).

[3] D. Hestenes, M. Wells, and G. Swackhamer, Force Concept Inventory, Phys. Teach. 30, 141 (1992).

[4] R. K. Thornton and D. R. Sokoloff, Assessing student learning of Newton's laws: The Force and Motion Conceptual Evaluation and the evaluation of active learning laboratory and lecture curricula, Am. J. Phys. 66, 338 (1998).

[5] R. R. Hake, Interactive-engagement versus traditional methods: A six-thousand-student survey of mechanics test data for introductory physics courses, Am. J. Phys. 66, 64 (1998).

[6] S. Freeman, S. L. Eddy, M. McDonough, M. K. Smith, N. Okoroafor, H. Jordt, and M. Pat. Wenderoth, Active learning increases student performance in science, engineering, and mathematics, Proc. Natl. Acad. Sci. U.S.A. 111, 8410 (2014).

[7] Aaron Adair, Student misconceptions about Newtonian mechanics: Origins and solutions through changes to instruction, Ph.D. thesis, The Ohio State University, 2013.

[8] R. D. Bock, Estimating item parameters and latent ability when responses are scored in two or more nominal categories, Psychometrika 37, 29 (1972).

[9] Physport, https://www.physport.org. Accessed 10/31/ 2020.

[10] Table II for the Force Concept Inventory (revised from 081695R), http://modeling.asu.edu/R\&E/FCI-RevisedTableII_2010.pdf. Accessed 3/17/2019.

[11] L. Crocker and J. Algina, Introduction to Classical and Modern Test Theory (Holt, Rinehart and Winston, New York, 1986).

[12] M. Planinic, L. Ivanjek, and A. Susac, Rasch model based analysis of the Force Concept Inventory, Phys. Rev. Phys. Educ. Res. 6, 010103 (2010).

[13] J. Wang and L. Bao, Analyzing Force Concept Inventory with item response theory, Am. J. Phys. 78, 1064 (2010).

[14] J. Han, L. Bao, L. Chen, T. Cai, Y. Pi, S. Zhou, Y. Tu, and K. Koenig, Dividing the Force Concept Inventory into two equivalent half-length tests, Phys. Rev. Phys. Educ. Res. 11, 010112 (2015).

[15] T. F. Scott and D. Schumayer, Students' proficiency scores within multitrait item response theory, Phys. Rev. Phys. Educ. Res. 11, 020134 (2015).

[16] Á. Pérez-Lemonche, J. Stewart, B. Drury, R. Henderson, A. Shvonski, and D. E. Pritchard, Mining Students Pre-instruction Beliefs for Improved Learning, in Proceedings of the Sixth (2019) ACM Conference on Learning @ Scale (ACM, Chicago IL USA, 2019), pp. 1-10.

[17] A. Traxler, R. Henderson, J. Stewart, G. Stewart, A. Papak, and R. Lindell, Gender fairness within the Force Concept Inventory, Phys. Rev. Phys. Educ. Res. 14, 010103 (2018).

[18] J. Stewart, C. Zabriskie, S. DeVore, and G. Stewart, Multidimensional item response theory and the Force Concept Inventory, Phys. Rev. Phys. Educ. Res. 14, 010137 (2018).

[19] P. Eaton and S. Willoughby, Identifying a preinstruction to postinstruction factor model for the Force Concept Inventory within a multitrait item response theory framework, Phys. Rev. Phys. Educ. Res. 16, 010106 (2020).

[20] P. Eaton, K. Johnson, and S. Willoughby, Generating a growth-oriented partial credit grading model for the Force Concept Inventory, Phys. Rev. Phys. Educ. Res. 15, 020151 (2019).

[21] T. I. Smith, K. J. Louis, B. J. Ricci IV, and N. Bendjilali, Quantitatively ranking incorrect responses to multiplechoice questions using item response theory, Phys. Rev. Phys. Educ. Res. 16, 010107 (2020).

[22] G. A. Morris, L. Branum-Martin, N. Harshman, S. D. Baker, E. Mazur, S. Dutta, T. Mzoughi, and V. McCauley, Testing the test: Item response curves and test quality, Am. J. Phys. 74, 449 (2006).

[23] G. A. Morris, N. Harshman, L. Branum-Martin, E. Mazur, T. Mzoughi, and S. D. Baker, An item response curves analysis of the Force Concept Inventory, Am. J. Phys. 80, 825 (2012).

[24] M. Reckase, Multidimensional Item Response Theory, Statistics for Social and Behavioral Sciences (Springer, New York, NY, 2009). 
[25] G. A. Morris, P. Walter, S. Skees, and S. Schwartz, Transition matrices: A tool to assess student learning and improve instruction, Phys. Teach. 55, 166 (2017).

[26] L. Viennot, Spontaneous reasoning in elementary dynamics, Eur. J. Sci. Educ. 1, 205 (1979).

[27] Lillian C. McDermott, Research on conceptual understanding in mechanics, Phys. Today 37, No. 7, 24 (1984).

[28] I. A. Halloun and D. Hestenes, Common sense concepts about motion, Am. J. Phys. 53, 1056 (1985).

[29] J. Clement, D. E. Brown, and A. Zietsman, Not all preconceptions are misconceptions: Finding 'anchoring conceptions' for grounding instruction on students' intuitions, Int. J. Sci. Educ. 11, 554 (1989).

[30] D. Hestenes, Toward a modeling theory of physics instruction, Am. J. Phys. 55, 440 (1987).

[31] E. Brewe, J. Bruun, and I. G. Bearden, Using module analysis for multiple choice responses: A new method applied to Force Concept Inventory data, Phys. Rev. Phys. Educ. Res. 12, 020131 (2016).

[32] J. Wells, R. Henderson, J. Stewart, G. Stewart, J. Yang, and A. Traxler, Exploring the structure of misconceptions in the Force Concept Inventory with modified module analysis, Phys. Rev. Phys. Educ. Res. 15, 020122 (2019).

[33] J. Yang, J. Wells, R. Henderson, E. Christman, G. Stewart, and J. Stewart, Extending modified module analysis to include correct responses: Analysis of the Force Concept Inventory, Phys. Rev. Phys. Educ. Res. 16, 010124 (2020).

[34] A. A. DiSessa, Toward an epistemology of physics, Cognit. Instr. 10, 105 (1993).

[35] A. A. Disessa and B. L. Sherin, What changes in conceptual change?, Int. J. Sci. Educ. 20, 1155 (1998).

[36] M. T. H. Chi and J. D. Slotta, The ontological coherence of intuitive physics, Cognit. Instr. 10, 249 (1993).

[37] M. T. H. Chi, J. D. Slotta, and N. De Leeuw, From things to processes: A theory of conceptual change for learning science concepts, Learning Instr. 4, 27 (1994).

[38] J. D. Slotta, M. T. H. Chi, and E. Joram, Assessing students' misclassifications of physics concepts: An ontological basis for conceptual change, Cognit. Instr. 13, 373 (1995).

[39] J. Minstrell, Facets of students' knowledge and relevant instruction, in Research in Physics Learning: Theoretical Issues and Empirical Studies, edited by R. Duit, F. Goldberg, and H. Niedderer (IPN, Kiel, Germany, 1992), pp. 110-128.
[40] D. Hammer, Misconceptions or p-prims: How may alternative perspectives of cognitive structure influence instructional perceptions and intentions, J. Learn. Sci. 5, 97 (1996).

[41] D. Hammer, More than misconceptions: Multiple perspectives on student knowledge and reasoning, and an appropriate role for education research, Am. J. Phys. 64, 1316 (1996).

[42] D. Hammer, Student resources for learning introductory physics, Am. J. Phys. 68, S52 (2000).

[43] M. Chi and R. Roscoe, The processes and challenges of conceptual change, in Reconsidering Conceptual Change: Issues in Theory and Practice, edited by M. Limon and L. Mason (Kluwer Academic Publishers, New York, 2002), pp. 3-27.

[44] L. Bao and E. Redish, Model analysis: Representing and assessing the dynamics of student learning, Phys. Rev. Phys. Educ. Res. 2, 1 (2006).

[45] US News \& World Report: Education, https://premium .usnews.com/best-colleges. Accessed 5/28/2020.

[46] See Supplemental Material at http://link.aps.org/ supplemental/10.1103/PhysRevPhysEducRes.17.010122 for item response curves for each sample, for density plots for samples 2 and 3, and for dendrograms and skree plots for all samples.

[47] J. Wells, R. Henderson, A. Traxler, P. Miller, and J. Stewart, Exploring the structure of misconceptions in the Force and Motion Conceptual Evaluation with modified module analysis, Phys. Rev. Phys. Educ. Res. 16, 010121 (2020).

[48] L. Hu and P. M. Bentler, Cutoff criteria for fit indexes in covariance structure analysis: Conventional criteria versus new alternatives, Struct. Equ. Modeling 6, 1 (1999).

[49] A. E. Raftery, Bayesian model selection in social research, Sociol. Methodol. 25, 111 (1995).

[50] T. F. Scott, D. Schumayer, and A. R. Gray, Exploratory factor analysis of a Force Concept Inventory data set, Phys. Rev. Phys. Educ. Res. 8, 020105 (2012).

[51] M. R. Semak, R. D. Dietz, R. H. Pearson, and C. W. Willis, Examining evolving performance on the Force Concept Inventory using factor analysis, Phys. Rev. Phys. Educ. Res. 13, 010103 (2017).

[52] Y. Suh and D. M. Bolt, Nested logit models for multiplechoice item response data, Psychometrika 75, 454 (2010). 